\title{
Reconfigurable Multilevel Phase-Shift Keying Encoder-Decoder for All-Optical Networks
}

\author{
M. R. Mokhtar, M. Ibsen, P. C. Teh, and D. J. Richardson
}

\begin{abstract}
We demonstrate the application of a uniform Bragg grating as a dynamically reconfigurable phase encoder-decoder for optical systems. Precise discrete phase modulation between chips is obtained simply by heating segments along the grating with fine resistive wires. Its reliability to generate and recognize various phase code sequences is demonstrated in a 16-chip 20-Gchip/s quaternary phase-shift keying coherent optical code-division multiple access experiment. The bit-error-rate response is also included to highlight its performance.
\end{abstract}

Index Terms-Code-division multiplexing, gratings, matched filters, optical fiber communication.

\section{INTRODUCTION}

$\mathbf{O}$ PTICAL MULTIPLEXING techniques have been adopted to cater to higher speed and bandwidth requirements of future optical networks. Wavelength-division multiplexing (WDM) and time-division multiplexing (TDM) have been successfully deployed. However, these conventional techniques generate spectrally inefficient systems through dedication of one wavelength per user and require strict temporal synchronization of the networks, respectively. The depletion of the available frequencies in the transmission band of an optical fiber calls for more appropriate multiplexing techniques. Recently, optical code-division multiple access (OCDMA) has been identified as an alternative technique to solve the future wavelength resource problems. OCDMA can be overlaid onto the existing WDM networks to enhance network versatility. Moreover, it allows flexible bandwidth management, asynchronous operation, improved system security, and the potential for much higher connectivity [1].

OCDMA systems have advanced to a stage where 255-chip systems have been experimentally demonstrated, utilizing superstructured fiber Bragg grating (SSFBG) technology [2]. Nevertheless, definite advancement of the optical transmission systems to dynamically reconfigurable networks brings about the need for this multiplexing system to include tuning capabilities. Recently, we proposed a practical dynamically reconfigurable encoder-decoder based on a uniform fiber Bragg grating (FBG) to suit this requirement [3]. The continuous phase-shifting property of this device can be further explored to enhance the capacity of a highly flexible optical network through multilevel phase-shift keying operation.

Manuscript received July 12, 2002; revised November 12, 2002.

The authors are with the Optoelectronics Research Centre, University of Southampton, Highfield, Southampton SO17 1BJ, U.K. (e-mail mrm@orc.soton.ac.uk).

Digital Object Identifier 10.1109/LPT.2003.807902
In this letter, we have simplified the construction of the device configuration and increased its code sequence generation, and demonstrate its application in a 16-chip 20-Gchip/s quaternary phase-shift keying (QPSK) OCDMA experiment. Autocorrelation and cross correlation signatures are presented to show the reliability of code sequence generation and recognition of the device. We also present its bit-error-rate (BER) measurements to support its applicability in data transportation.

\section{DEVICE PRINCIPLE AND DESCRIPTION}

Advances in fiber grating fabrication technology enables precise control of the grating amplitude and spatial phase. A discrete phase shift is typically achieved by shifting the phase of the rapidly varying refractive index during the grating inscription process. This has been successfully exploited for phase encoding of data signals in OCDMA systems [2]. On the other hand, a confined chirp will also produce similar effect as a result of the change in the local propagation constant. The chirp can be caused either by variation in the grating period or the effective refractive index, following the phase matching condition. In fact, both variations occur when the temperature of the grating is elevated, as dictated by the second term of the following [4]:

$$
\begin{aligned}
\Delta \lambda_{B}=2\left(\Lambda \frac{\delta n_{\mathrm{eff}}}{\delta l}+n_{\mathrm{eff}}\right. & \left.\frac{\delta \Lambda}{\delta l}\right) \Delta l \\
& +2\left(\Lambda \frac{\delta n_{\mathrm{eff}}}{\delta T}+n_{\mathrm{eff}} \frac{\delta \Lambda}{\delta T}\right) \Delta T
\end{aligned}
$$

where $\Delta \lambda_{B}$ is the shift in the Bragg grating center wavelength, $n_{\text {eff }}$ is the effective refractive index of the fiber core, $\Lambda$ is the grating period, $\Delta l$ is the length expansion, and $\Delta T$ is the temperature change. However, the contribution of the thermo-optic effect is greater than the thermo-elastic effect in silica. Moreover, the induced effective index variation is impermanent as long as the temperature does not exceed the grating erasure temperature $\left(\sim 150^{\circ} \mathrm{C}\right)$. Apparently, a fine electric conductor with an applied voltage can adequately provide the required heat and can thus be used to induce a controllable chirp.

Based on this concept, a phase encoder with multiple tunable phase shifts can be formed from a single uniform FBG. The local phase profile in this device is altered by varying the temperature along the grating according to a desired code sequence. In this demonstration, a uniform FBG is written using our continuous grating writing technique operated with continuous wave ultraviolet light at $244 \mathrm{~nm}$ [5]. The grating is $80 \mathrm{~mm}$ long and is written in a standard telecom compatible fiber with a numerical aperture (NA) of approximately 0.12 . It has also been preannealed at a temperature that exceeds $200{ }^{\circ} \mathrm{C}$ to de- 


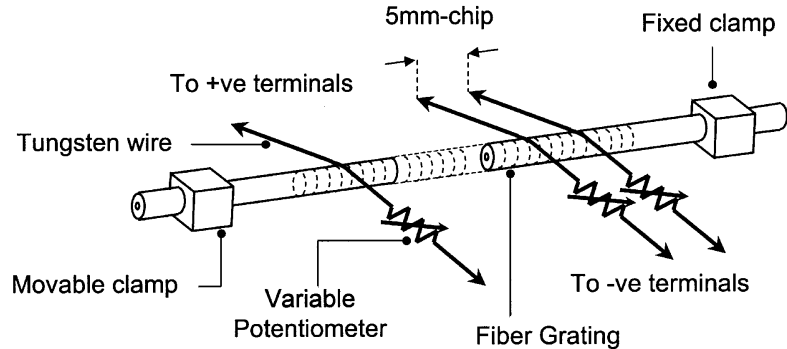

Fig. 1. Schematic structure of the reconfigurable encoder-decoder.

liberately weaken the strength and consequently becomes very stable when operating below this temperature. The peak reflectivity of the fiber grating is then about $57 \%$ corresponding to a uniform coupling coefficient in the grating of approximately $12 \mathrm{~m}^{-1}$. Low reflectivity ensures signal light to penetrate its full length and also the coded pulse sequences will have the same amplitude.

A length of tungsten wire (18- $\mu \mathrm{m}$ diameter), in series with a variable potentiometer is laid across the fiber to provide an adjustable localized heating. A 16-chip encoder-decoder with a chip duration of $50 \mathrm{ps}$ is thus constructed by positioning 15 parallel wires $5 \mathrm{~mm}$ apart along the fiber grating with the first wire being placed $5 \mathrm{~mm}$ into the grating (Fig. 1). Additionally, the fiber grating is mounted on a stretcher to allow tuning of the grating central wavelength. The values of electrical current required to induce the desired phase shifts to the back-reflected light are predetermined through observation of the spectral dynamic behavior of the grating being heated in the center [3]. Recognizing that the phase shift is in fact proportional to the product of the heated length and the associated Bragg wavelength modulation, it is expected to roughly show linear dependence with the applied current, as the heated region is extremely narrow. The values of electrical current used to generate $0.5 \pi$, $\pi$, and $1.5 \pi$ are found to be 30,60 , and $90 \mathrm{~mA}$, respectively. The highest operating temperature of the wires is thus measured to be only around $27^{\circ} \mathrm{C}$ above the room temperature.

\section{OCDMA SYSTEM EXPERIMENT AND RESULTS}

In order to test the application of our proposed reconfigurable encoder-decoder, a simple OCDMA system is constructed. The experimental setup is shown in Fig. 2. The transmitter consists of a tunable laser operated at $1548 \mathrm{~nm}$, an electroabsorption modulator (EAM), an electrooptic modulator (EOM), and a reconfigurable optical encoder (as described above). The EAM, driven with a $10-\mathrm{GHz}$ sinusoidal signal is used to convert the continuous light from the tunable laser to a $10-\mathrm{GHz}$ pulse train with the full-width at half-maximum (FWHM) of 20 ps. The pulse train is then modulated by an EOM at a data rate of $622 \mathrm{Mb} / \mathrm{s}$ in response to the electrical data from a pseudorandom pattern generator (PPG). After phase-encoding performed by the reconfigurable encoder, the encoded signal stream is diverted into two arms of a $3-\mathrm{dB}$ coupler, each having a unique four-level phase-encoded superstructured grating. Subsequently, the reflected pulses in each arm are observed on an oscilloscope and an error detector.

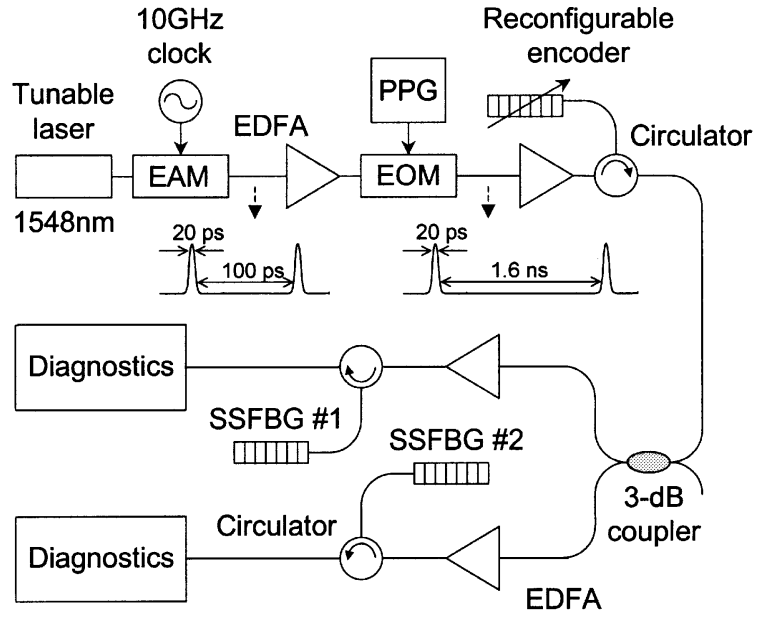

Fig. 2. Experimental setup.
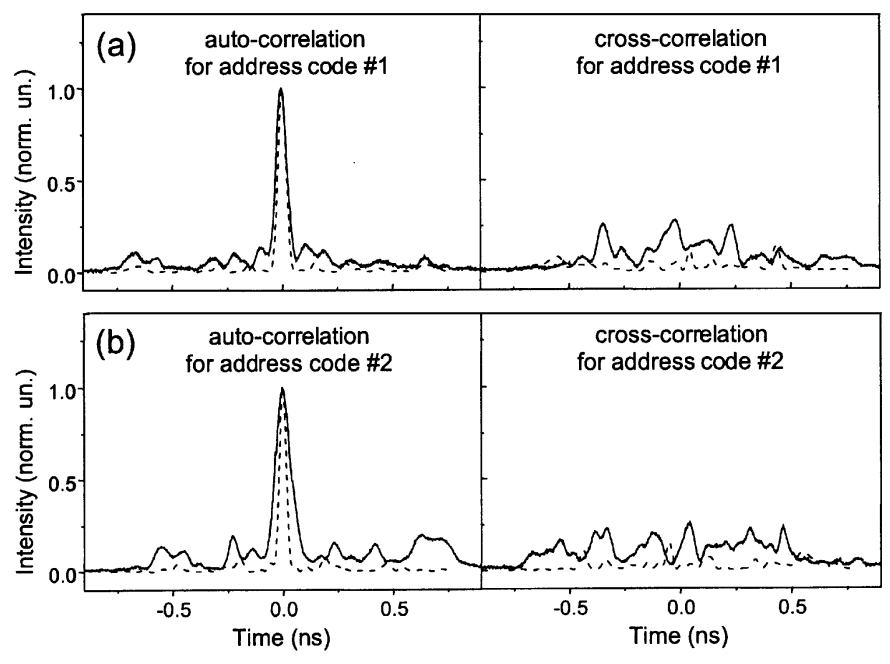

Fig. 3. Traces of the intensity auto and cross correlation (solid lines: measured; dashed lines: calculated) for (a) address code \#1 and (b) address code \#2.

The optical encoding is performed in the time domain, a procedure that is known as the direct sequence OCDMA. Each signal pulse is directly transformed into a coded pulse sequence upon reflection from the reconfigurable encoder. The device is set to the inverse code sequence with respect to either one of the SSFBG decoders so that a time-reversal operation can be performed. The reflected pulses are configured to a desired address code by independently adjusting the electrical current flow at the chip boundaries to their associated values. The 16-chip QPSK coded pulse stream is individually matched filtered by the SSFBGs at the receiver, both operating at the same central wavelength of $1548 \mathrm{~nm}$. Fig. 3(a) shows the relative intensity distribution of a bit of the decoded signal in the matched and unmatched cases for the SSFBG with address code \#1 ( $\pi, \pi, \pi$, $0.5 \pi, 0.5 \pi, 1.5 \pi, 0.5 \pi, 1.5 \pi, \pi, 0,0.5 \pi, 1.5 \pi, \pi, 1.5 \pi, 0,0)$. On the other hand, Fig. 3(b) represents that of the SSFBG with address code \#2 $(1.5 \pi, 1.5 \pi, 0.5 \pi, 0, \pi, 0.5 \pi, 1.5 \pi, 0.5 \pi, 0,1.5 \pi$, $0, \pi, 1.5 \pi, 0.5 \pi, 1.5 \pi)$. These codes belong to the Family $\mathcal{A}$ sequences [6]. The overall extinction ratio between auto and cross correlation is found to be approximately $71 \%$. 


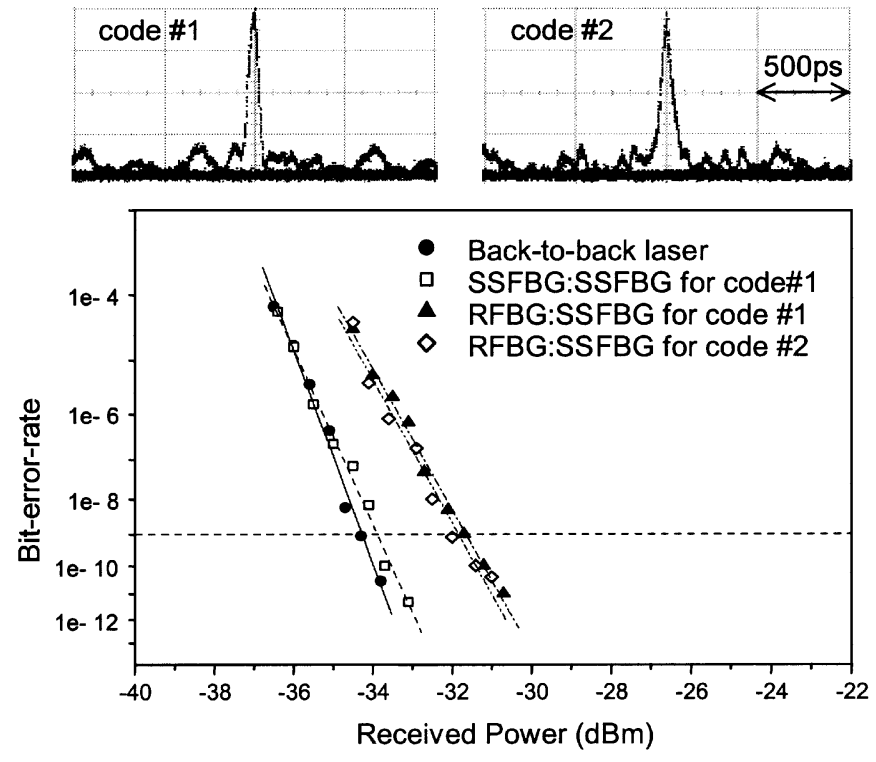

Fig. 4. BER curves of the $622 \mathrm{Mb} / \mathrm{s}$ signals (RFBG: reconfigurable encoder; SSFBG: fixed-code decoder). Inset shows eye diagrams of the decoded signals for address code \#1 (left) and address code \#2 (right).

The thermal distribution is believed to resemble the shape of hyperbolic secant. Consequently, the analytical spectral response of the device can be readily obtained by introducing gradual phase shift at the chip boundaries using the transfer matrix models. The Fourier transform of the product of the resulting complex reflection coefficients with the spectral response of the SSFBG (discrete phase shift) represents the auto/cross correlation. These results are overlaid with their corresponding experimental time responses in Fig. 3. Extended simulation analysis reveals that the autocorrelation intensity is slightly less in the case of match filtering process that employs a reconfigurable encoder, relative to that using only SSFBGs. It may stem from the fact that the phase shift is not totally discrete as it is distributed over a finite length. This prediction is confirmed by the BER measurements for the two address codes, shown in Fig. 4. A power penalty of approximately $2.5 \mathrm{~dB}$ with respect to the laser back-to-back response is observed.
Minor uncertainties of the current values for the phase shifts of interest may also attribute to this measured value. Nevertheless, good autocorrelations (Fig. 3) and eye diagrams (inset in Fig. 4) measured in this demonstration, strongly justify its practical implementation. This also indicates high tolerance of the device to offsets in the phase shifts. In another independent empirical observation, the switching interval between code reconfiguration is found to be only a matter of seconds in a temperature-controlled environment.

\section{CONCLUSION}

We have demonstrated the application of a reconfigurable encoder-decoder in a 16-chip 20-Gchip/s QPSK coherent OCDMA system. Our results verify the possibility of channel expansion through exploitation of the continuous phase-tuning capability of our proposed device. Good auto/cross correlation obtained through match filtering with a phase-encoded superstructured fiber grating indicates very precise dynamic code sequence generation and recognition by the device. BER measurements also show excellent data transportation.

\section{REFERENCES}

[1] N. Wada and K. Kitayama, "A $10 \mathrm{~Gb} / \mathrm{s}$ optical code-division multiplexing using 8-chip optical bipolar code and coherent detection," $J$. Lightwave Technol., vol. 17, pp. 1758-1765, Oct. 1999.

[2] P. C. Teh, M. Ibsen, J. H. Lee, P. Petropoulos, and D. J. Richardson, "Demonstration of a four-channel WDM/OCDMA system using 255-chip, 320 Gchip/s quaternary phase coding gratings," IEEE Photon. Technol. Lett., vol. 14, pp. 227-229, Feb. 2002.

[3] M. R. Mokhtar, M. Ibsen, P. C. Teh, and D. J. Richardson, "Simple dynamically reconfigurable OCDMA encoder/decoder based on a uniform fiber Bragg grating," in Proc. OFC'2002, Anaheim, CA, 2002, Paper ThGG54, pp. 688-690.

[4] A. Othonos and K. Kalli, Fiber Bragg Grating: Fundamentals and Applications in Telecommunications and Sensing. Norwood, MA: Artech House, 1999, pp. 95-147.

[5] M. Ibsen, M. K. Durkin, M. J. Cole, M. N. Zervas, and R. I. Laming, Recent Advances in Long Dispersion Compensating Fiber Bragg Gratings. London, U.K.: IEE, 1999.

[6] S. Boztas, R. Hammons, and P. V. Kumar, "4-Phase sequence with nearoptimum correlation properties," IEEE Trans. Inform. Theory, vol. 38, pp. 1101-1113, May 1992. 